\title{
DISTRIBUCIÓN DE ARSÉNICO EN AGUA SUPERFICIAL Y SEDIMENTO EN LA CUENCA DEL RÍO CARRIZAL, MANABÍ - ECUADOR
}

\author{
Ana María Aveiga Ortiz ${ }^{* a}$, Patricio Javier Noles Aguilara ${ }^{\mathrm{a}}$, Fabián Peñarrieta Macías ${ }^{\mathrm{a}}$, \\ Erika Murgueitio Herrerab
}

\begin{abstract}
RESUMEN
En diversas fuentes naturales de agua superficial de Ecuador, así como en otras a nivel mundial, la concentración de arsénico supera los límites permisibles $(10 \mu \mathrm{g} / \mathrm{L})$ establecidos por la Organización Mundial de la Salud. El objetivo de la presente investigación fue determinar los niveles de arsénico en agua superficial y sedimentos en la cuenca del río Carrizal (Manabí, Ecuador), zona que posee una importante actividad agropecuaria. Se monitorearon 21 estaciones en tres zonas, cubriendo una extensión de $51 \mathrm{~km}$. Las concentraciones de arsénico en agua y sedimento se evaluaron mediante espectrometría de absorción atómica con el equipo PerkinElmer® AAnalyst ${ }^{\mathrm{TM}} 200$, de acuerdo con los lineamientos del Standard Methods Edición 2015, por la metodología APHA AWWA WEF 3114-B. Se utilizó el diseño experimental de bloques al azar, con arreglo factorial de las tres zonas de muestreo. Los resultados indican que se encontraron concentraciones de arsénico de hasta 35,64 mg/L en agua superficial y de $47,41 \mathrm{mg} / \mathrm{kg}$ en sedimentos. Se concluye que estos altos niveles de arsénico en la cuenca del río Carrizal podrían ser de origen antrópico.
\end{abstract}

Palabras clave: microcuenca, subcuenca, embalse La Esperanza, contaminación.

\section{ARSENIC DISTRIBUTION IN SUPERFICIAL WATER AND SEDIMENT AT THE CARRIZAL RIVER BASIN, MANABI-ECUADOR}

\begin{abstract}
In various natural sources of surface water in Ecuador, as well as in others worldwide, the concentration of arsenic exceeds the permissible limits $(10 \mu \mathrm{g} / \mathrm{L})$ established by the World Health Organization. The objective of these investigation was to determine the levels of arsenic in surface water and sediments in the Carrizal river basin (Manabí, Ecuador), an area

\footnotetext{
a Escuela Superior Politécnica Agropecuaria de Manabí, Carrera de Ingeniería Ambiental, Calceta, Manabí, Ecuador, aaveiga@espam.edu.ec

b Universidad de las Fuerzas Armadas - ESPE, Departamento de Ciencias de la Tierra y la Construcción
} 
that has an important agricultural activity. 21 stations in three areas were monitored, covering an area of $51 \mathrm{~km}$. Arsenic concentrations in water and sediment were evaluated by means of atomic absorption spectrometry with the PerkinElmer ${ }^{\circledR}$ AAnalyst TM 200 kit, in accordance with the guidelines of Standard Methods Edition 2015, by the APHA AWWA WEF 3114B methodology. The experimental design of random blocks was applied, with a factorial arrangement of the three sampling areas. The indicated results are found for arsenic of up to $35,64 \mathrm{mg} / \mathrm{L}$ in surface water and 47,41 mg / $\mathrm{kg}$ in sediments. It is concluded that these high levels of arsenic in the Carrizal river basin could be of anthropogenic origin.

Key words: Micro basin, sub-basin, La Esperanza Reservoir, contamination.

\section{INTRODUCCIÓN}

Actualmente, se estima que más de un millón de especies contaminantes son introducidas en fuentes de agua a través de vertidos antropogénicos; de allí que, los estudios de calidad de las cuencas hidrográficas han adquirido gran interés en las últimas décadas dado el incremento de la población en sus riberas, el creciente grado de industrialización y aportes del sector primario ${ }^{1}$. Los metales pesados como el arsénico (As) en aguas y sedimentos se caracterizan por ser extremadamente tóxicos, altamente persistentes y de rápida acumulación por los organismos ${ }^{2,3}$. En México, Argentina, Chile, Perú y Nicaragua, el arsénico (As) en el ambiente y en las fuentes de agua para consumo humano es debido a factores geológicos; aunque, las actividades antropogénicas como explotación minera, refinación de metales por fundición, procesos electrolíticos para producción de metales como cadmio y zinc también contribuyen a incrementar el contenido de este metal en el ambiente ${ }^{4}$.

Las formas solubles del As como el ácido metilarsónico (MMA) y el ácido dimetilarsónico (DMA) son fuertemente tóxicas, la ingestión de dosis elevadas causa problemas gastrointestinales, cardiovasculares, disfunciones del aparato nervioso, y finalmente a la muerte ${ }^{1,5}$. En general, las formas inorgánicas son más tóxicas que las orgánicas, y el arsenito es más peligroso que el arsenato ${ }^{6}$. En Argentina, se detectaron niveles de As en aguas subterráneas entre 20 y $4600 \mu \mathrm{g} / \mathrm{L}$. Además, en el mismo país se constató que durante el periodo 2010-2016, tanto para los efectos carcinogénicos y no carcinogénicos, las concentraciones de As presentan alto riesgo al superar los umbrales máximos en la población de Las Flores?.

En Ecuador, se ha encontrado que el contenido de elementos riesgosos, entre ellos As, en aguas, sedimentos y peces en la cuenca del río Santiago (Esmeraldas), superan los valores permisibles, con rangos de afectación biológica en la mayoría de las estaciones de muestreo; exhibiendo niveles de contaminación moderados ${ }^{8}$. En función de la problemática generada por la presencia de metales pesados, específicamente As, y ante la imperativa necesidad de plasmar un plan de gestión para el manejo de fuentes hídricas, se presenta el caso del río Carrizal (Manabí-Ecuador), de donde se exponen resultados sobre la presencia y distribución de arsénico tanto en el agua superficial como en sedimentos. 


\section{PARTE EXPERIMENTAL}

\section{1. Área de muestreo}

El estudio se realizó en la cuenca hidrográfica del río Carrizal (Cantón Bolívar, Manabí); cuenca comprendida entre las coordenadas $14^{\prime} 15^{\prime \prime} \mathrm{S}, 79$ 52'12" $\mathrm{W}$, con un área de aproximadamente $1390 \mathrm{~km}^{2}$, la cual se ubica en la provincia Manabí y limita al norte con las cuencas del río Briceño y río Jama, al sur con las cuencas del río Portoviejo y río Guayas, al este con el Océano Pacífico y la cuenca del Estero Pajonal y, al oeste con la cuenca del río Guayas?.

De acuerdo a las características hidrológicas, geológicas, climatológicas, actividades antropogénicas y de uso del suelo, y a criterios de identificación, accesibilidad y representatividad del objetivo de estudio, se establecieron 21 estaciones de monitoreo a lo largo de la subcuenca del río Carrizal, distribuidas de la siguiente manera: 7 estaciones en la microcuenca (17 km de extensión), 5 estaciones en el embalse La Esperanza (8 km de extensión) y 9 estaciones distribuidas en $26 \mathrm{~km}$ en la subcuenca del río Carrizal, con lo cual se cubrió una extensión total de aproximadamente $51 \mathrm{~km}$ (figura 1). El estudio se dividió en las siguientes zonas:

- Zona 1 (Microcuenca): Severino, Balsa en Medio, Tigre Adentro, Río Chico, Puente Carrizal, El Frutal.

- Zona 2 (Embalse La Esperanza): Carrizal, Bejuco, Dos Bocas, Membrillo, Embalse La Esperanza

- Zona 3 (Subcuenca): Quiroga, La Esperanza, Barranco Colorado, Sarampión, Mata Palo, Los Almendros, San Bartolo, La Karina, Limón.

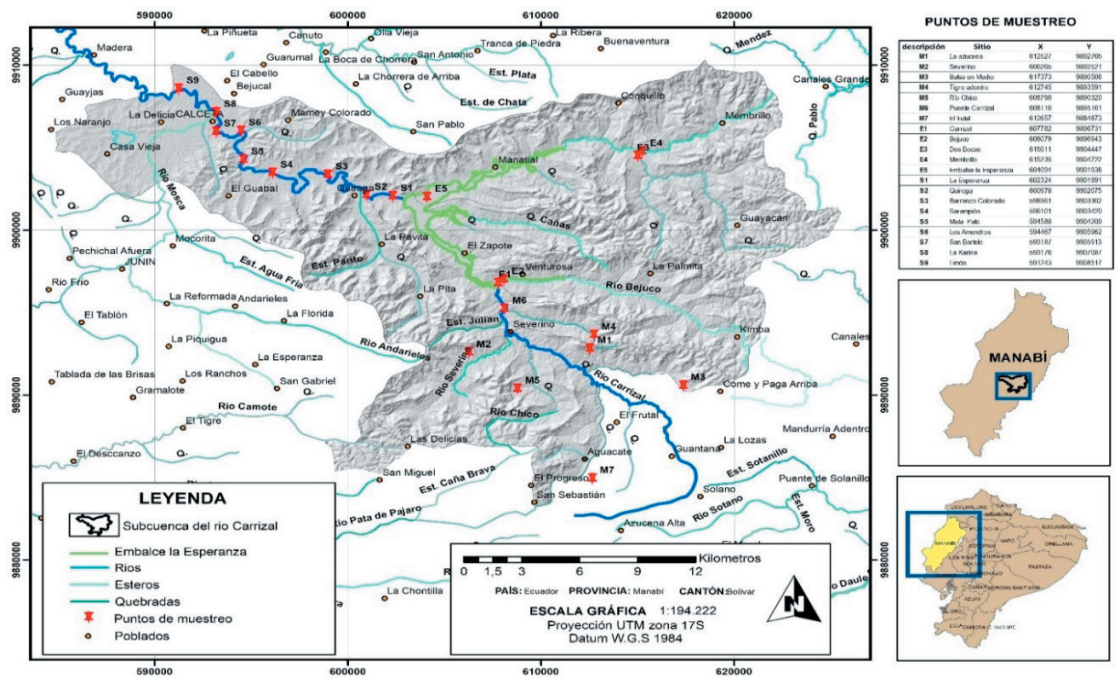

Figura 1. Croquis de la cuenca hidrográfica del río Carrizal en la República del Ecuador. 


\section{Toma de muestras de aguas y sedimentos}

De acuerdo al régimen unimodal de distribución de las precipitaciones de la zona en estudio, los muestreos se realizaron dos veces al año, durante los meses de agosto y octubre, correspondientes a un periodo de caudal medio y al caudal de estiaje, respectivamente ${ }^{9}$. El protocolo para toma, conservación y manipulación de las muestras de agua, se tomó como referencia a UNE-EN ISO 5667-3:2019 y a Murgueitio et al. (2018), con adaptaciones de acuerdo a las necesidades del proyecto. Se tomó una muestra superficial, lo más cercano al centro del cuerpo de agua y en contra de la corriente al flujo del recurso hídrico; estas muestras fueron colectadas con una botella muestreadora de fondo horizontal ${ }^{10}$. Para el análisis de arsénico, las muestras fueron acidificadas con $\mathrm{HNO}_{3} 0,1 \mathrm{~N}$ con $\mathrm{pH}$ de 3,0 a 4,0 y refrigeradas a $4,0^{\circ} \mathrm{C}$ para su conservación desde el momento de la recolección ${ }^{11}$.

Para la recolección de las muestras de sedimentos se tomó como referencia a Murgueitio et al. (2018), y en cada estación de muestreo se tomó aproximadamente un kilogramo de sedimento superficial a la profundidad de $25-50 \mathrm{~cm}$, colectados desde la orilla del río, con un barreno; colocadas en bolsas plásticas con cierre hermético, y conservadas a $4,0^{\circ} \mathrm{C}^{10}$. En el Embalse La Esperanza, la recolección se realizó a una profundidad de 7 a $33 \mathrm{~m}$ con el apoyo de una draga tipo Van Veen de acero inoxidable, lanzada desde una barca ${ }^{10}$.

\section{Determinación de arsénico}

Los análisis fueron realizados en el Laboratorio de Medio Ambiente, Departamento de Ciencias de la Tierra y de la Construcción de la Universidad de las Fuerzas Armadas (ESPE).

\section{Equipos, materiales y reactivos}

\section{a. Equipos y materiales}

a) Espectrómetro de absorción atómica Perkin Elmer ${ }^{\circledR}$ AAnalyst ${ }^{\mathrm{TM}}$ 200, con generador de hidruros (límite de detección de $0,15 \mu \mathrm{g} / \mathrm{L}$ ) equipado con el quemador para llama de aireacetileno y con la celda de cuarzo colocada en el soporte del quemador.

b) Lámpara de As de cátodo hueco

\section{b. Reactivos}

a) Ácido clorhídrico $(0,200 \mu \mathrm{g} / \mathrm{L})$, b) Hidróxido de sodio $(0,25 \mathrm{~mol} / \mathrm{L}$, c) Tetrahydroborato de sodio (0,8 mol/L), d) Argón, disponible comercialmente. e) Solución de Ioduro de Potasio: Disolver 3 g de KI y 5 g de L(+)-Ácido ascórbico en $100 \mathrm{~mL}$ de agua desionizada, b) Soluciones de As. La solución stock contiene $1000 \mathrm{mg} / \mathrm{L}$ de As.

Para elaborar las curvas de calibración, se utilizó una réplica, con un tiempo de integración de diez (10) segundos.

\section{c. Descripción}

El arsénico es convertido en hidruro mediante reacción con el borohidruro de sodio y se transporta dentro de atomizador de absorción atómica. Los hidruros son depurados continuamente con argón dentro de la celda de cuarzo calentada por la llama del espectrómetro de absorción atómica y convertidos a átomos en fase gaseosa. 


\section{d. Condiciones recomendadas para el uso del equipo Perkin Elmer AA200}

Estas condiciones son dadas por el manual de uso del equipo, para el As.

Longitud de onda 193,70 nm; ranura $0,7 \mathrm{~nm}$; ruido relativo 1; concentración característica $0,1 \mu \mathrm{g} / \mathrm{L}$; concentración característica de chequeo $5 \mu \mathrm{g} / \mathrm{L}$; rango lineal a $5 \mu \mathrm{g} / \mathrm{L}$; flujo de oxidante $10 \mathrm{~L} / \mathrm{min}$ Arsénico flujo de acetileno 3,3 L/min.

\section{Análisis estadístico}

Se utilizó el diseño experimental completamente aleatorizado, con arreglo factorial de los tratamientos, donde los factores correspondieron a las zonas de muestreo (microcuenca, embalse y subcuenca) y los meses (agosto y octubre), mientras que las repeticiones estuvieron representadas por las estaciones de muestreo.

Previo al análisis de varianza (ANOVA), los valores de las características cuantificadas relacionadas a las concentraciones de As tanto en agua como en sedimentos fueron examinados por las pruebas de normalidad de Wilk-Shapiro y la de homogeneidad de varianza de Bartlett por el programa ASSISTAT Versión $7.7(2016)^{12}$. Hubo necesidad de transformar los datos en raíz cuadrada de $(\mathrm{X}+1)$ para satisfacer supuestos del ANOVA. Todos los análisis se realizaron con el software estadístico InfoStat ${ }^{\circledR}{ }^{13}$.

\section{RESULTADOS Y DISCUSIÓN}

Para la Zona 1 (microcuenca que consta de los puntos de monitoreo Severino, Balsa en Medio, Tigre Adentro, Río Chico, Puente Carrizal, El Frutal) se encontró que las concentraciones de As en sedimento fueron menores a $0,001 \mathrm{mg} / \mathrm{kg}$ y en agua van desde no detectable por el equipo hasta $23,05 \mathrm{mg} / \mathrm{L}$ (figura 2). Resultados similares se obtuvieron en las provincias de Guayas y Los Ríos (Ecuador) donde las concentraciones de As disuelto (en aguas de riego, ríos y de pozo) fueron inferiores a $10 \mu \mathrm{g} / \mathrm{L}$; sin embargo, en el sitio de Taura, los valores oscilaron entre 12 y $19 \mu \mathrm{g} / \mathrm{L}^{14}$. 


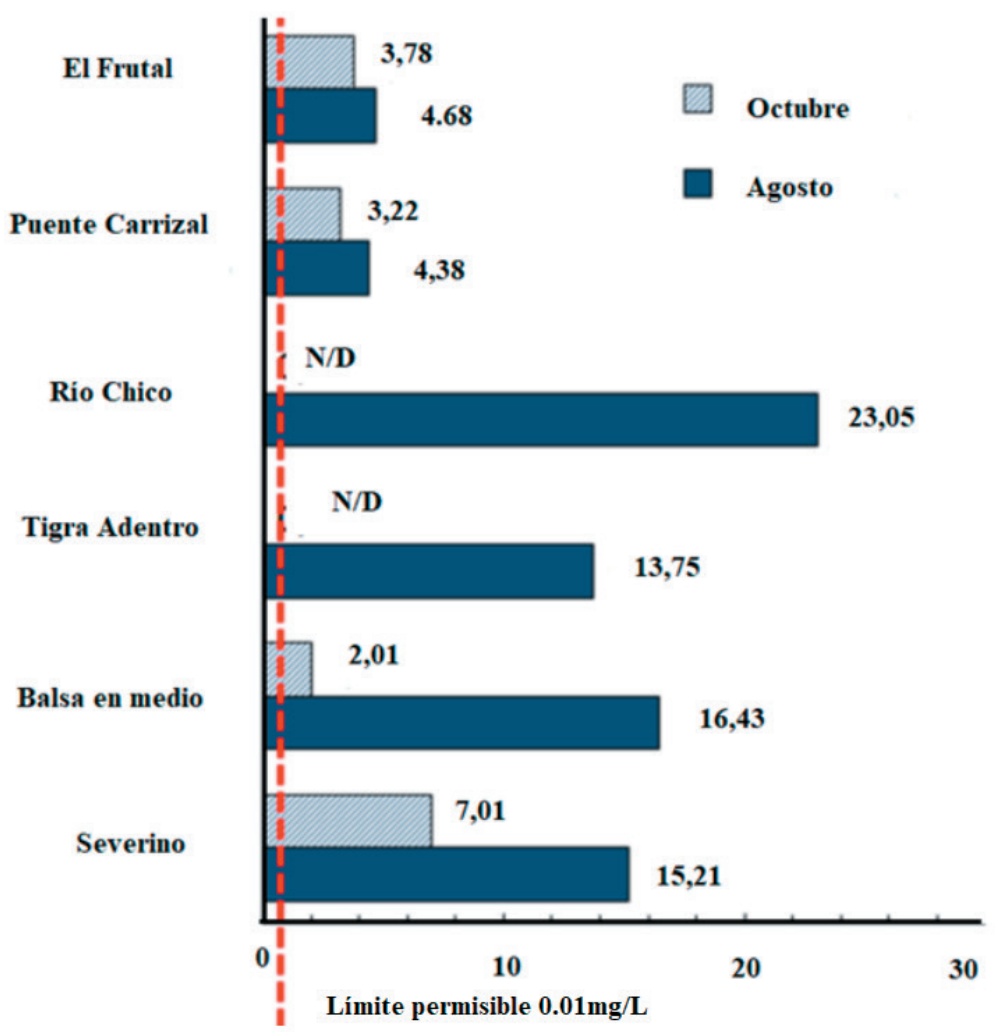

Figura 2. Zona 1 (microcuenca): variación de las concentraciones de arsénico en $\mu \mathrm{g} / \mathrm{L}$ en agua durante los meses de muestreo.

Concentraciones relativamente bajas de arsénico disuelto en agua también sugieren una atenuación del arsénico en sedimentos más profundos, lo cual se atribuyó a cambios en las condiciones REDOX a lo largo de la cuenca asociada a la planicie Chianan (Taiwán) y a las interacciones con el Mn y el Fe depositado en el perfil de sedimentos, que funciona como una barrera de atenuación ${ }^{15}$.

Para la Zona 2 (Embalse La Esperanza: Carrizal, Bejuco, Dos Bocas, Membrillo, Embalse La Esperanza) se presentan las concentraciones de As en la figura 3, siendo los niveles en el sedimento menores al límite detectable por el equipo, y en agua varían desde no detectable por el equipo hasta $20,11 \mathrm{mg} / \mathrm{L}$. El As interactúa con diferentes tipos de sedimentos y condiciones ambientales a lo largo de los ríos y arroyos; por lo tanto, el As acuoso y extraíble de los sedimentos depende de la ubicación geográfica, en el norte de Ecuador, los ríos de El Ángel, San Pedro y Pichan y Quebradas Cachiyacu y Rumihuaycu tienen un contenido de arsénico muy diverso de $974 \mu \mathrm{g} / \mathrm{L}$ a $2 \mu \mathrm{g} / \mathrm{L}^{16}$. 


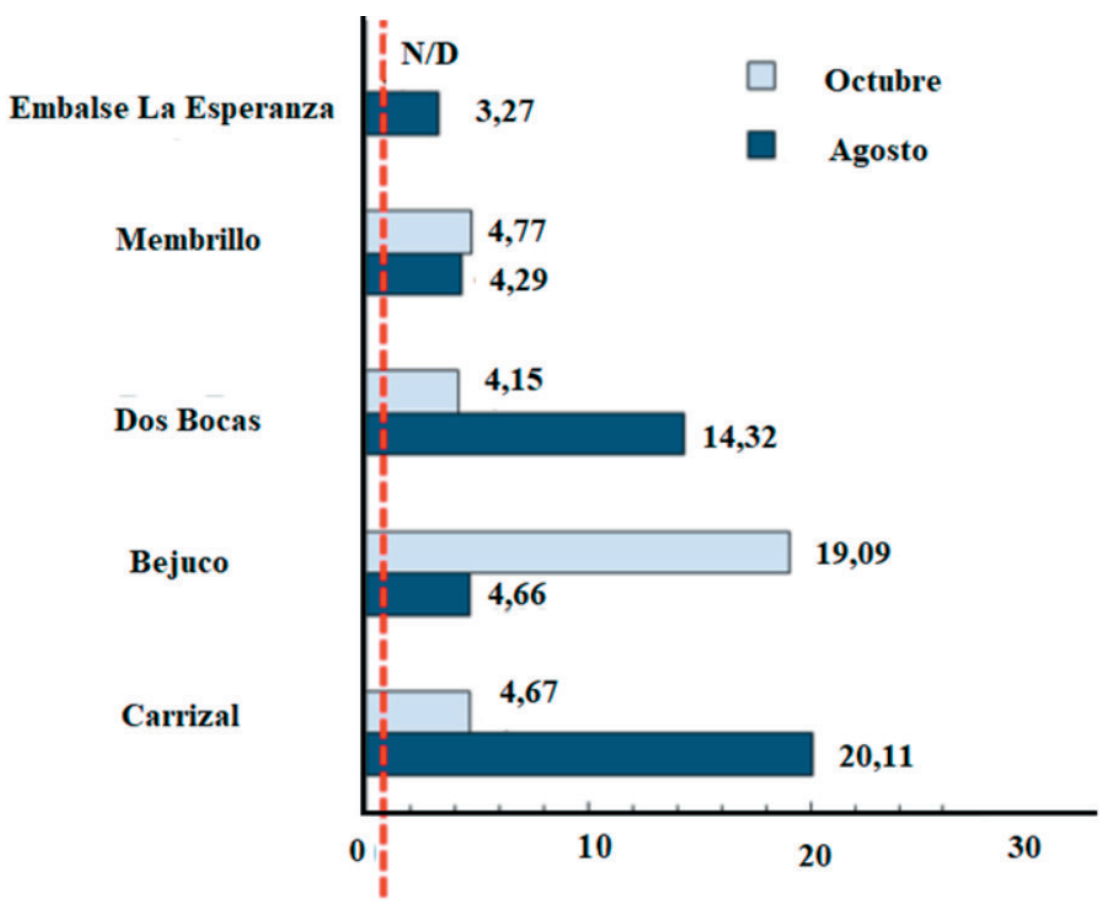

Límite permisible $0.01 \mathrm{mg} / \mathrm{L}$

Figura 3. Zona 2 (Embalse La Esperanza): variación de las concentraciones de arsénico en $\mu \mathrm{g} / \mathrm{L}$ en agua durante los meses de muestreo.

Para la Zona 3 (Subcuenca: Quiroga, La Esperanza, Barranco Colorado, Sarampión, Mata Palo, Los Almendros, San Bartolo, La Karina, Limón) se encontró los niveles de As en agua y sedimento que se muestran en la figura 4. Las concentraciones en agua llegan a un máximo de $35,64 \mathrm{mg} / \mathrm{L}$ y en sedimentos hasta $47,41 \mathrm{mg} / \mathrm{kg}$. En la laguna Limoncocha - Ecuador el agua no presenta valores significativos de As, pero en sedimentos hay concentración de hasta $1,3 \mathrm{mg} / \mathrm{kg}$ de As, por lo que probablemente este elemento debe estar en una forma química que dificulta su solubilidad ${ }^{17}$. 

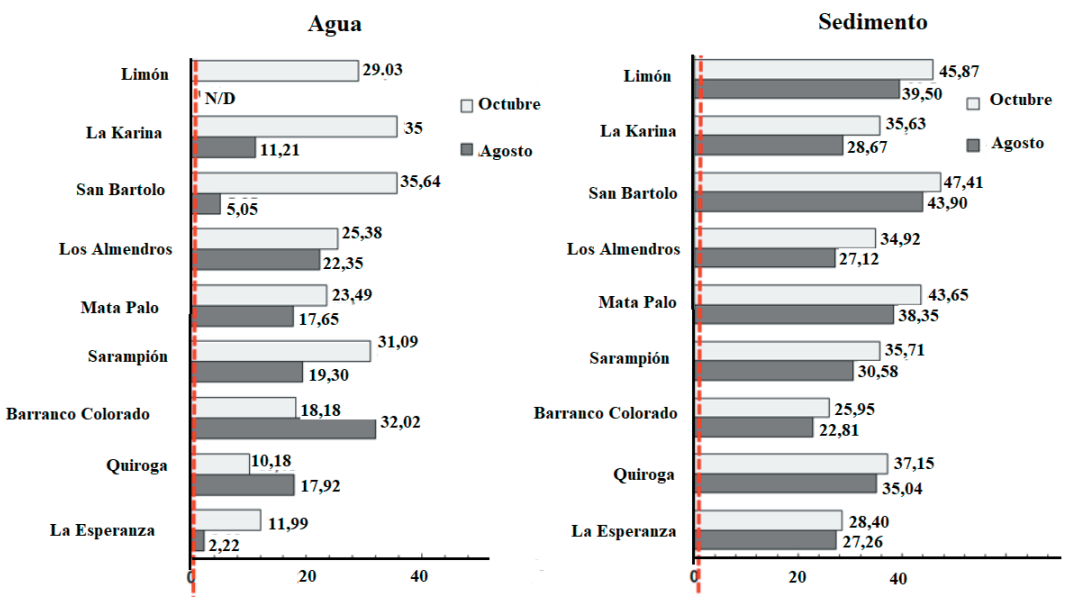

Figura 4. Zona 3 (Subcuenca): variación de las concentraciones de arsénico en ppm tanto en agua y sedimento durante dos meses de muestreo.

Se reporta el coeficiente de distribución del arsénico entre el sedimento y el agua superficial para las tres zonas de monitoreo. Este coeficiente fue calculado en base a las concentraciones de As en la fase sólida (sedimento) y en la fase líquida (agua superficial), según la siguiente ecuación:

$$
\mathrm{Kd}=\frac{\text { Concentración Arsénico en sedimiento }(\mathrm{mg} / \mathrm{kg})}{\text { Concentración Arsénico en agua }(\mathrm{mg} / \mathrm{L})}
$$

Valores relativamente bajos de $\mathrm{Kd}$ indican que el arsénico tiene mayor grado de disolución en la fase acuosa (figura 5); mientras que, valores altos de $\mathrm{K}_{\mathrm{d}}$ indican mayor preferencia del arsénico por la fase sólida (sedimento). La movilización o distribución del arsénico está sujeta a cambios y transformaciones biogeoquímicas, así como a la desorción en acuíferos, debido a cambios de $\mathrm{pH}$ o reacciones REDOX. Además, existe asociación con actividades microbianas, que inducen un ambiente reductor. También, la distribución o partición del arsénico se debe a la desorción por competencia iónica con fosfatos, nitratos, carbonatos y silicatos $^{18,19,20,21,22}$.

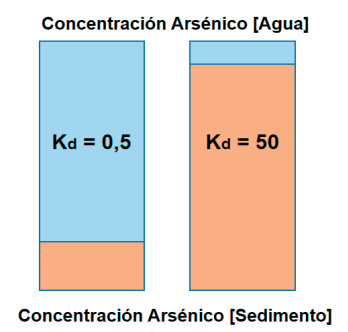

Figura 5. Coeficiente de distribución del arsénico en agua superficial y sedimento. 
En la figura 6, según los resultados de caracterización para la Zona 1 -Microcuenca- dado los valores bajos del coeficiente de partición $\mathrm{K}_{\mathrm{d}}$, el arsénico tiene mayor disolución en el agua superficial que preferencia por el sedimento, para los dos meses monitoreados. Este efecto ya ha sido reportado ${ }^{23}$, en estudios de la cuenca del Río Rojo que nace en las montañas de la provincia de Yunnan, en China y discurre hacia el sudeste atravesando el territorio norte de Vietnam, en donde que las concentraciones más altas de arsénico ocurrieron en la transición de la temporada de lluvias a la estación seca y las más bajas al final de la estación seca, lo cual se debe a variaciones en las condiciones redox debido a niveles fluctuantes de las aguas subterráneas que indujeron la liberación de As a la fase líquida a través de mecanismos reductores u oxidativos. Las variaciones a largo plazo y estacionales en las concentraciones de As en el agua tienen implicaciones importantes con fines epidemiológicos, ya que sirven de referencia para la reducción de incertidumbre en la estimación de riesgos a la salud por exposición al contaminante y para diseñar estrategias de mitigación de impactos a través del descarte de aguas de mayor riesgo y explotación de recursos hídricos donde las concentraciones se mantengan en niveles aceptables ${ }^{24}$.

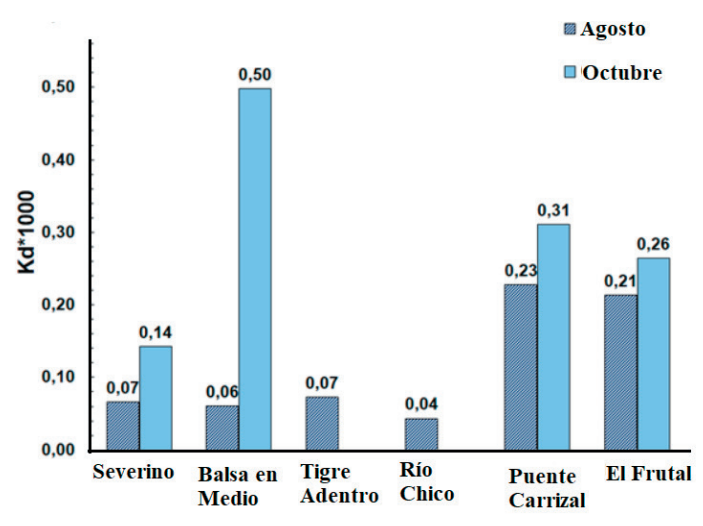

Figura 6. Coeficiente de distribución sedimento-agua para la Zona 1 (Microcuenca) de las concentraciones de arsénico durante dos meses de muestreo.

La caracterización para la Zona 2 -Embalse- indica que el As tiene mayor disolución en el agua superficial que preferencia por el sedimento, asimismo, para los dos meses monitoreados, dado los valores bajos del coeficiente de partición $\mathrm{K}_{\mathrm{d}}$, salvo en el mes de octubre en el punto de muestreo Embalse La Esperanza. Es decir, en el mes de octubre en este punto del embalse la concentración de arsénico fue mayor en el sedimento que en la muestra de agua superficial, en contraste con los otros puntos de monitoreo. 


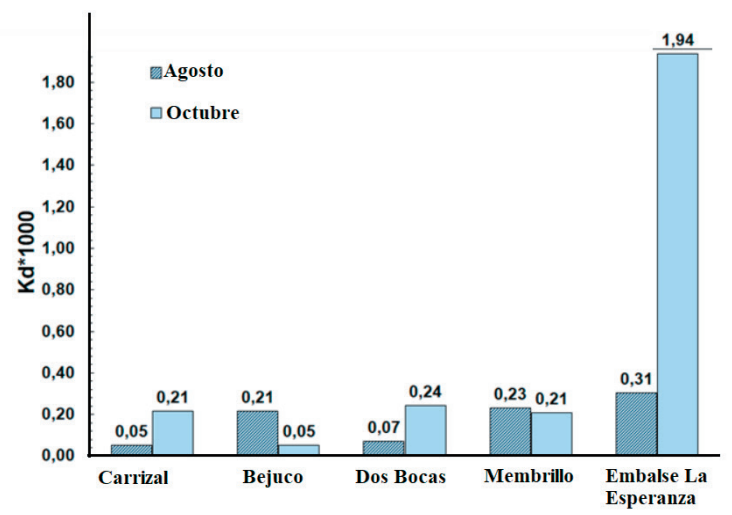

Figura 7. Coeficiente de distribución para la Zona 2 (Embalse La Esperanza) de las concentraciones durante dos meses de muestreo.

En la subcuenca del río Carrizal (Zona 3), el mayor coeficiente de distribución de la concentración de arsénico en los sedimentos, fue en el sector de La Esperanza y de San Bartolo durante los meses de monitoreo, como se indica en la figura 8.

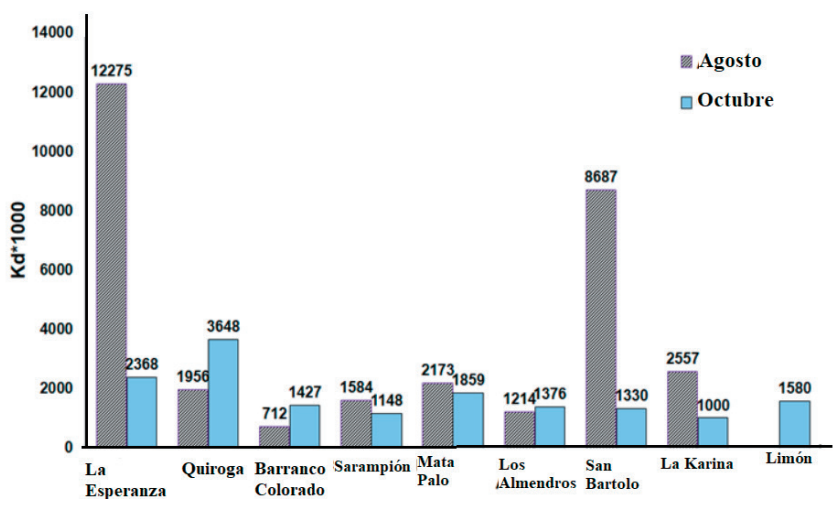

Figura 7. Coeficiente de distribución para la Zona 3 (Subcuenca) de las concentraciones de arsénico durante los meses de muestreo.

Se determinó la diferencia estadística entre dos variables: zona de muestreo (Zonas 1, 2 y 3), y los meses de muestreo (agosto y octubre) para las muestras de agua y sedimento, respectivamente (figuras 9 y 10). En las tres zonas evaluadas de la cuenca del río Carrizal, durante el mes de agosto no mostraron diferencias estadísticas entre sí; sin embargo, durante el mes de octubre, los niveles de As en la Zona 3 (Subcuenca) fueron superiores estadísticamente a los cuantificados en la Zona 2 (Embalse) y la Zona 1 (Microcuenca), los cuales resultaron similares entre sí. Las concentraciones encontradas de As no responden a un origen geológico, tal como se ha encontrado en otros estudios ${ }^{4}$; por ende, se afianza la hipótesis de que el arsénico aquí reportado procede de deposiciones antrópicas de la zona. 


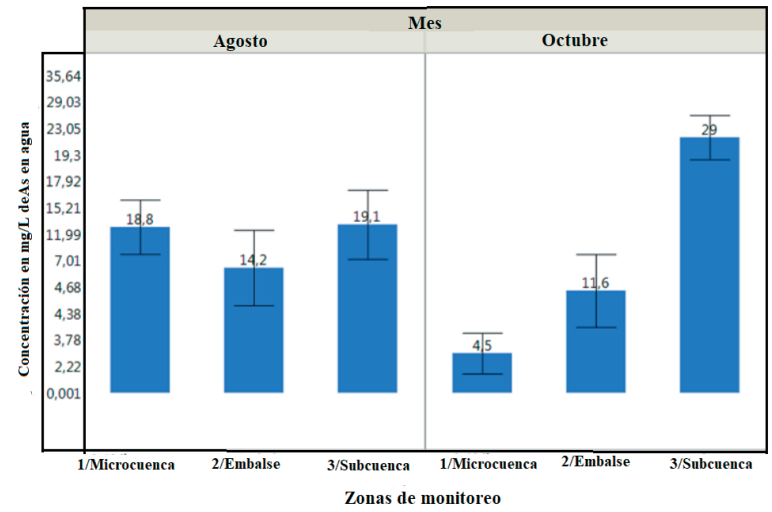

Figura 9. Variaciones de las concentraciones de arsénico en el agua superficial en tres zonas y dos meses de muestreo en la cuenca del río Carrizal (barras de error corresponden al nivel crítico de Tukey a $5 \%$ de probabilidad).

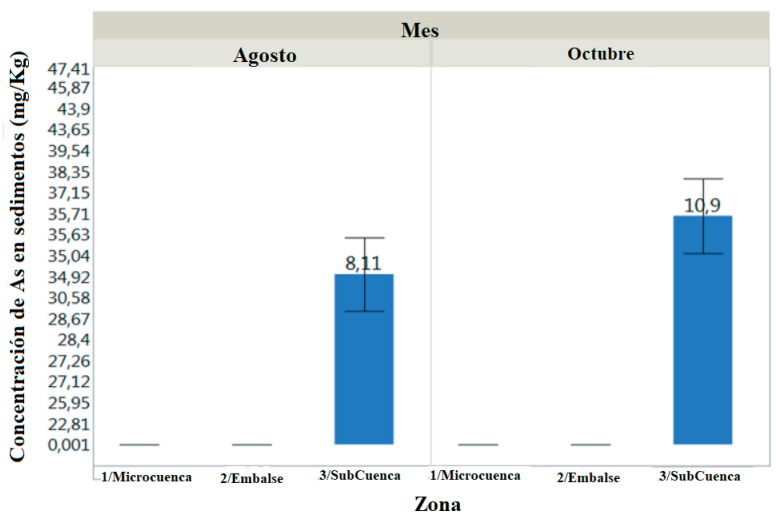

Figura 10. Variaciones de las concentraciones de arsénico en el sedimento en tres zonas y dos meses de muestreo en la cuenca del río Carrizal (barras de error corresponden al nivel crítico de Tukey a $5 \%$ de probabilidad).

De acuerdo al análisis de varianza (tabla 1), se observaron diferencias altamente significativas para las tres zonas de muestreo, tanto para la concentración de As en agua como en sedimento. De manera similar, se verificó diferencia significativa para la interacción entre las dos variables estudiadas (zona de muestreo y meses de muestreo). La comparación de las concentraciones de As en sedimentos por la prueba de Tukey a 5\% de probabilidad (tabla 2) indica que a nivel de la Zona 3 (Subcuenca) los valores promedio de arsénico fueron estadísticamente superiores a los obtenidos tanto para la Zona 1 (Microcuenca) como para la Zona 2 (Embalse La Esperanza), siendo que estos dos últimos son similares entre sí. 
Tabla 1. Resumen del análisis de varianza para la variable concentraciones de arsénico en sedimentos y aguas en la cuenca del río Carrizal.

\begin{tabular}{|c|c|c|c|c|c|}
\hline \multirow{3}{*}{$\begin{array}{l}\text { Fuente de } \\
\text { Variación }\end{array}$} & \multirow{3}{*}{ Gl } & \multicolumn{4}{|c|}{ Cuadrados medios } \\
\hline & & \multicolumn{2}{|c|}{ Datos observados } & \multicolumn{2}{|c|}{ Datos transformados $(\mathrm{X}+1)^{0,5}$} \\
\hline & & Sedimentos & Aguas & Sedimentos & Aguas \\
\hline Zona & 2 & $6023,66^{* *}$ & $758,76^{* *}$ & $121,77^{* *}$ & $14,57 * *$ \\
\hline Meses & 1 & 22,16 & 20,54 & 0,15 & 1,78 \\
\hline Zonas x Meses & 2 & 26,21 & $329,45 * *$ & 0,18 & $7,23 * *$ \\
\hline Error & 34 & 24,22 & 61,47 & 0,17 & 1,12 \\
\hline $\mathrm{CV}(\%)$ & & 31,35 & 58,28 & 12,76 & 30,17 \\
\hline
\end{tabular}

** Diferencias altamente significativas $(\mathrm{p}<0,01)$

Tabla 2. Comparaciones de los valores promedios de arsénico en sedimentos en tres zonas de la cuenca del río Carrizal.

\begin{tabular}{lccc}
\hline \multirow{2}{*}{ Zona } & \multicolumn{2}{c}{ Concentraciones de arsénico } & \multirow{2}{*}{$\begin{array}{c}\text { Ámbito } \\
\text { estadístico }\end{array}$} \\
\cline { 2 - 3 } & Observados & Transformados & A \\
\hline Subcuenca & 34,885 & 5,96 & $\mathrm{~B}$ \\
Microcuenca & 0,001 & 1,00 & $\mathrm{~B}$ \\
Embalse & 0,001 & 1,00 &
\end{tabular}

Medias seguidas de la misma letra no difieren entre sí (Tukey a $5 \%$ de probabilidad)

Se han identificado cinco mecanismos naturales de contaminación de fuentes naturales debido al arsénico: actividad volcánica o hidrotermal; meteorización oxidativa de sulfuros arsénicos, típicamente pirita y arseno-pirita; desorción de As desde superficies minerales a $\mathrm{pH}$ alto; reducción de oxi-hidróxidos de hierro sedimentario y concentración evaporativa ${ }^{16}$. La presencia de As en aguas de consumo humano en ciertos ríos de México, Nicaragua, Perú, Argentina y Chile e incluso Ecuador ${ }^{5,6,8,16}$ se atribuye a factores geológicos; sin embargo, en el caso de la cuenca del Río Carrizal, no se han reportado ninguno de estos procesos en forma natural, por lo cual se presume que la fuente de contaminación de aguas y sedimentos en la región tenga origen antrópico. A nivel de la Zona 3 (Subcuenca del rio Carrizal), donde se encontraron niveles más altos de As en agua, es conocido que para las localidades de La Esperanza, Quiroga, Barranco Colorado, Sarampión, Mata Palo y los Almendros existe contaminación del suelo y presión sobre los recursos naturales por actividades agropecuarias; mientras que, para San Bartolo, La Karina y El Limón, la contaminación del agua es ocasionada por establecimientos humanos y, en el caso particular de la localidad El Limón se 
agregan actividades agropecuarias ejercidas por una empresa bananera. Por lo que es posible que dentro de esas localidades se empleen agroquímicos en el manejo de las actividades agropecuarias y sean ellos los que generen parte o el total de los niveles de contaminación constatada por As en la subcuenca de Carrizal.

La comparación de las variaciones en las concentraciones de arsénico en las estaciones de muestreo en función de los meses, realizada a través de la prueba de t pareada, (tabla 3) no mostró diferencias significativas para las concentraciones de As en muestras de agua superficial; no obstante, sí se encontraron diferencias significativas para los niveles de As en sedimentos, obteniéndose mayores concentraciones durante el muestreo de octubre que corresponde a la época de caudal de estiaje del río Carrizal.

Tabla 3. Variaciones estacionales de las concentraciones de arsénico en aguas y sedimentos en diferentes estaciones de muestreo en la cuenca del río Carrizal.

\begin{tabular}{lcccccc}
\hline Variable & Agosto & Octubre & $\begin{array}{c}\text { Diferencia } \\
\text { promedio }\end{array}$ & $\begin{array}{c}\text { Desviación } \\
\text { estándar }\end{array}$ & t & Probabilidad \\
\hline As en agua & 13,41 & 13,49 & $-0,08 \mathrm{~ns}$ & 14,10 & $-0,03$ & 0,980 \\
As en sedimentos & 14,66 & 16,73 & $-2,07 * *$ & 2,77 & $-3,34$ & 0,003 \\
\hline
\end{tabular}

${ }^{(* *)}$ Significativo a $1 \%$ de probabilidad por la prueba de $\mathrm{t}$ pareada.

En relación a los valores de los límites de los concentraciones de arsénico para las normas canadiense, europea y ecuatoriana (tabla 4), se observa que los valores de arsénico en aguas para las zonas y meses muestreados de la cuenca del río Carrizal superan los límites establecidos en las tres normas consideradas; sin embargo, para los sedimentos en las dos meses de muestreo únicamente a nivel de la subcuenca del río Carrizal es donde los valores de arsénico superan los límites establecidos de acuerdo a la norma canadiense. No existe referencia de límite de concentraciones de arsénico en sedimentos ni para la norma europea ni para la ecuatoriana. 
Tabla 4. Comparación de las concentraciones de arsénico en aguas y sedimentos de la cuenca del río Carrizal en relación a referencias internacionales.

\begin{tabular}{|c|c|c|c|c|}
\hline \multirow{3}{*}{ Zona de muestreo } & \multicolumn{4}{|c|}{ Concentraciones de arsénico } \\
\hline & \multicolumn{2}{|c|}{ Aguas (mg/L) } & \multicolumn{2}{|c|}{ Sedimentos $(\mathrm{mg} / \mathrm{kg})$} \\
\hline & Agosto & Octubre & Agosto & Octubre \\
\hline Microcuenca & 12,917 & 2,669 & 0,001 & 0,001 \\
\hline Embalse & 9,330 & 6,639 & 0,001 & 0,001 \\
\hline Subcuenca & 16,005 & 24,514 & 32,584 & 37,186 \\
\hline \multicolumn{5}{|c|}{ Norma ambiental de referencia } \\
\hline Canadiense $^{1}$ & \multicolumn{2}{|c|}{0,005} & \multicolumn{2}{|c|}{$5, \overline{900}$} \\
\hline Europea $^{2}$ & \multicolumn{2}{|c|}{0,010} & & \\
\hline Ecuatoriana $^{3}$ & \multicolumn{2}{|c|}{0,100} & & \\
\hline
\end{tabular}

Canadiense $^{1}=$ Canadian Council of Ministers of the Environment. Europea ${ }^{2}$ : Criterios de calidad de aguas continental de la directiva 75/440/EEC de la Unión Europea; Ecuatoriana ${ }^{3}=$ Ministerio del Ambiente, Edición Especial, Año III, Nº 387, noviembre 2015.

\section{CONCLUSIONES}

La determinación de las concentraciones de As en zonas agropecuarias, hace imprescindible la georreferenciación para el conocimiento en futuras decisiones ambientales y evitar la biomagnificación de este metal. En este sentido, se concluye que las concentraciones de As en sedimentos de la subcuenca del río Carrizal fueron estadísticamente superiores a los niveles de la microcuenca y en el embalse; mientras que las mayores concentraciones en el agua superficial se presentaron en la subcuenca en el mes de octubre. Las concentraciones de As en los sedimentos superaron los límites nacionales e internacionales establecidos para el consumo humano y para la preservación de la vida acuática, obteniéndose los valores más altos durante la época de caudal de estiaje del río Carrizal. No se han reportado fuentes naturales de arsénico en la cuenca del río Carrizal, por lo que se presume que su presencia es de origen antrópico, lo cual amerita estudios adicionales para su confirmación.

\section{AGRADECIMIENTO}

Un cordial agradecimiento a Carlos Banchón (Universidad Agraria del Ecuador) por su asesoría en la determinación del Coeficiente de Distribución del As en agua superficial y sedimento. Un agradecimiento a la Escuela Superior Politécnica Agropecuaria de Manabí, Carrera de Ingeniería Ambiental, Calceta, Manabí, Ecuador por permitir el desarrollo de la investigación "Evaluación de los niveles de contaminación por metales pesados en la Microcuenca del Río Carrizal” del Grupo de Investigación "Gestión Integrada de Recursos Naturales y Biodiversidad para el Desarrollo Sostenible”. 


\section{REFERENCIAS BIBLIOGRÁFICAS}

1. Rosas H. Estudio de la contaminación por metales pesados en la cuenca del Llobregat [Tesis Doctoral]. Barcelona, España: Universitat Politècnica de Catalunya; 2001.

2. Smedley S, Kinniburg D. A review of the source, behavior and distribution of arsenic in natural waters. Appl Geochem. 2002; 17: 517-568.

3. Wang S, Mulligan C. Occurrence of arsenic contamination in Canada: Sources, behavior and distribution. Sci Total Environ. 2006; 366: 701-721.

4. Zambrano CR, González TL, Aranguré ZF, Espinosa RM, Paredes LJ. ¿Arsénico en Nayarit?. Revista Fuente. 2011; 3 (6): 25-30.

5. Reyes YC, Vergara I, Torres OE, Díaz MJ, González EE. Contaminación por metales pesados: Implicaciones en salud, ambiente y seguridad Alimentaria. Ingeniería Investigación y Desarrollo. 2016; 16 (2): 66-77.

6. Katzung B. Farmacología básica. México: Editorial Manual Moderno; 2001.

7. Mertens TF. Arsénico en el agua de consumo: riesgo y percepción del riesgo en Las Flores, Provincia de Buenos Aires [Tesis de pregrado]. Tandil: Universidad Nacional del Centro de la Provincia de Buenos Aires; 2018.

8. Correa CM, Bolaños OM, Rebolledo ME, Rubi, MD, Salinas RE. Análisis del contenido de metales en aguas, sedimentos y peces en la cuenca del río Santiago, Provincia de Esmeraldas, Ecuador. Investigación y Saberes. 2015; 4 (2): 32-42.

9. MAE: Ministerio del Ambiente de Ecuador. Proyecto adaptación al cambio climático a través de una efectiva gobernabilidad del agua en el Ecuador (PACC). Estudio de vulnerabilidad actual a los riesgos climáticos en el sector de los recursos hídricos en las cuencas de los Ríos Paute, Jubones, Catamayo, Chone, Portoviejo y Babahoyo. Quito: MAE; 2009.

10. Murgueitio E, Cumbal L, Abril M, Izquierdo A, Debut A, Tinoco O. Green Synthesis of Iron Nanoparticles: Application on the Removal of Petroleum Oil from Contaminated Water and Soils. J Nanotechnology. 2018: Article ID 4184769, 8 pages. doi:10.1155/2018/4184769

11. Prica M, Dalmacija B, Dalmacija M, Agbaba J, Krčmar D, Tricković J, Karlovic E. Changes in metal availability during sediment oxidation and the correlation with immobilization potential. Ecotoxicol Environ Saf. 2010; 73:1370- 1377.

12. Silva F, Azevedo C. The Assistat Software Version 7.7 and its use in the analysis of experimental data. Afr J Agric Res. 2016; 11(39): 3733-3740.

13. Di Rienzo J, Casanoves M, Balzarini L, González M, Robledo C. InfoStat versión 2017. Grupo InfoStat, FCA. Córdova, Argentina: Universidad Nacional de Córdoba; 2017.

14. Otero X, Tierra W, Atiaga O, Guanoluisa D, Nunes L, Ferreira T, Ruales J. Arsenic in rice agrosystems (water, soil and rice plants) in Guayas and Los Rios provinces, Ecuador. Sci Total Environ. 2016; 573: 778-787.

15. Su C, Zhu Y, Abbas Z, Huq M. Sources and controls for elevated arsenic concentrations in groundwater of Datong Basin, northern China. Environ Earth Sci. 2016; 75:1-13.

16. Cumbal L, Vallejo P, Rodríguez B, López D. Arsenic in geothermal sources at the northcentral Andean region of Ecuador: concentrations and mechanisms of mobility. Environ Earth Sci. 2010; 61:299-310. 
17. Coral K, Carrillo D, Oviedo J. Arsénico en aguas, suelos y sedimentos de la Reserva Biológica de Limoncocha - Ecuador con fines de conservación. INNOVA Res J. 2019; 4:158-169.

18. Rahman M, Siddiqi U, Kure S, Mano A, Udo K, Ishibashi Y. Mobilization of High Arsenic in the Shallow Groundwater of Kalaroa, South-Western Bangladesh. Expos Health. 2016; 8(2): 159-175.

19. Reza AS. Occurrence of arsenic in core sediments and groundwater in the ChapaiNawabganj District, northwestern Bangladesh. Water Res. 2010; 44: 2021-2037.

20. Xie X, Wang Y, Ellis A, Liu C, Duan M, Li J. Impact of sedimentary provenance and weathering on arsenic distribution in aquifers of the Datong basin, China: constraints from elemental geochemistry. J Hydrol. 2014; 519: 3541-3549.

21. Yu Q, Wang Y, Xie X, Ma R, Wu Y, Li J. Evaluation of groundwater flow model for arsenic mobilization in the Datong Basin Procedia. Earth Planetary Sci. 2013; 7: 924 927.

22. Berg M, Tran H, Nguyen C, Pham H, Schertenleib R, Giger W. Arsenic contamination of groundwater and drinking water in Vietnam: A human health threat. Environ Sci Technol. 2001; 35: 2621-2626.

23. Shahid M, Niazi N, Dumat C, Naidu R, Khalid S, Rahman M, Bibi I. A meta-analysis of the distribution, sources and health risks of arsenic-contaminated groundwater in Pakistan. Environ Pollut. 2018; 242: 307-319.

24. Ravenscroft P, Brammer H, Richards K. Arsenic pollution: a global synthesis. Oxford, United Kingdom: Wiley-Blackwell; 2009. 